\title{
CFD Modelling of Combustion and Heat Transfer in Compartment Fires
}

\author{
M.J. LEWIS, M.B. MOSS and P.A. RUBINI \\ School of Mechanical Engineering \\ Cranfield University \\ Bedford, England, UK
}

\begin{abstract}
The field modelling of compartment fires incorporates increasingly complex representations of the physical and chemical processes which may occur and these models, in turn, demand detailed evaluation. The influence of alternative descriptions of combustion in the fire source and the introduction of a ray tracing radiation model (discrete transfer) are assessed in this paper within the framework of progressively refined mesh geometry and by detailed comparison with the experimental measurements in the Steckler compartment fire. The predictions are made with SOFIE, a code specifically developed for building fire prediction. The reported comparisons demonstrate that detailed quantitative predictions of velocity and temperature fields can be performed accurately, given a satisfactory combination of grid resolution and physical modelling, particularly in relation to that of combustion and radiative exchange. Improved accuracy is shown to accompany the implementation of the discrete transfer radiation model, together with eddy break-up combustion, in comparison with the adoption of a more simply prescribed heat source. The introduction of a flamelet-based combustion model offers the additional prospect of incorporating more detailed chemistry, for example in relation to $\mathrm{CO}$, into room fire prediction.
\end{abstract}

KEYWORDS: Field Modelling ; Compartment Fires ; Combustion Modelling ; Radiation Heat Transfer.

\section{INTRODUCTION}

The wider application of field models to building fire simulation has naturally prompted concern for the accuracy of such detailed numerical predictions. The incorporation of increasingly complex models of the physical and chemical processes within the coupled system of partial differential equations describing a room fire, combined with elaborate numerical methods for their solution, poses particular challenges in relation to validation. Improvements in the representation of combustion in the fire source or of radiation heat 
transfer may be masked by inadequate spatial resolution of the phenomena involved or deficiencies in the numerical procedures implemented. Comparison with experimental measurement, on the other hand, is restricted to a small number of better-documented model fires. Even here the domain of interest is typically so large - for example, during the early stages of a compartment fire - that the experimental coverage is very limited. By its nature CFD evaluation is experimentally demanding and measurement campaigns undertaken for the assessment or development of less refined models - for example, incorporating zonal descriptions - are often of only limited value. The series of compartment fires investigated by Steckler et al [1] provides one of the more satisfactory test cases for field model prediction although the immediate vicinity of the fire source, where property changes are most pronounced, is also generally poorly resolved. A number of studies $[2,3,4,5]$ have used this compartment fire as a field model test case but have largely concentrated on flowfield characteristics. In common with other similar applications, combustion is often entirely omitted $[3,4,5]$ in favour of a simply prescribed heat source.

These same data are also used for model evaluation in the present paper. The investigation focusses, however, on the role of more detailed physical modelling, in particular, for radiative exchange by the discrete transfer method, including both properties modelling and numerical methods, the influence of the specific combustion model adopted and the opportunities for chemical species prediction, together with the contributions of mesh refinement and differencing scheme implemented.

\section{EXPERIMENTAL DETAIL}

These particular compartment fire experiments [1] centre on a square compartment, of side $2.8 \mathrm{~m}$ and height $2.18 \mathrm{~m}$, incorporating ceramic fibre board insulation and a circular gas burner, fuelled by commercial grade methane and having a diameter of $0.3 \mathrm{~m}$. The layout of the room is illustrated in fig. 1 . Of the several variations in burner position and ventilation configuration reported, the present paper concentrates on that in which the burner is positioned centrally in the room and ventilation is provided by a room opening (doorway) $1.83 \mathrm{~m}$ high by $0.74 \mathrm{~m}$ wide. The fuel flow rate selected corresponds to a heat output of 62.9 $\mathrm{kW}$. Conditions in the opening attracted particular attention and detailed measurements of temperature, using aspirated thermocouples, and velocity by bi-directional probe are reported there.

Figure 1.

Schematic Room Fire Compartment [1]

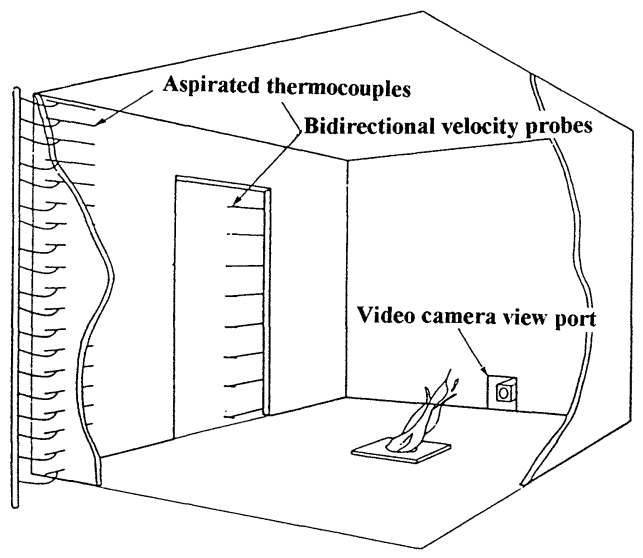




\section{NUMERICAL SIMULATION}

The simulation of this compartment fire has been performed using the code SOFIE

( Simulation of Fires In Enclosures ), specifically developed at Cranfield for room fire prediction. The code employs a general non-orthogonal coordinate system, with finite volume discretisation and a range of options for dependent variable interpolation

( Upwind, Hybrid, QUICK and TVD ). The SIMPLEC pressure correction algorithm is applied to the co-located velocities and pressure field. Turbulent closure is effected through a two-equation, k- $\varepsilon$ model incorporating buoyancy modifications [6].

Two combustion models have been implemented and compared here, based upon eddy break-up [7] and laminar flamelet [8] approaches, together with radiative exchange by the discrete transfer method [9].

\section{Combustion Modelling}

The eddy break-up combustion model assumes that the chemistry is fast and therefore turbulent mixing is rate controlling, according to the turbulence timescale $\mathrm{k} / \varepsilon$, such that the mass rate of fuel consumption may be written

$$
R_{f u}=\bar{\rho} \frac{\varepsilon}{k} \min \left[C_{R} m_{f u}, C_{R} m_{o x} / s, C_{R}^{\prime} m_{p r} /(1+s)\right]
$$

$\mathrm{m}_{\mathrm{fu}}, \mathrm{m}_{\mathrm{ox}}$ and $\mathrm{m}_{\mathrm{pr}}$ are the time-averaged mass fractions of fuel, oxidant and product respectively whilst $\mathbf{s}$ denotes the stoichiometric oxygen-to-fuel mass ratio. $\min [$ ] denotes the minimum of the composition arguments; $C_{R}$ and $C_{R}^{\prime}$, are empirically determined $\mathrm{O}(1)$ constants.

Together with a global, one-step description of methane combustion

$$
\mathrm{CH}_{4}+2 \mathrm{O}_{2} \rightarrow \mathrm{CO}_{2}+2 \mathrm{H}_{2} \mathrm{O}
$$

only one reactive scalar requires computation - for example, the fuel mass fraction, $\mathrm{m}_{\mathrm{fu}}$. The oxygen and product $\left(\mathrm{CO}_{2}\right.$ and $\left.\mathrm{H}_{2} \mathrm{O}\right)$ concentrations may then be determined from element conservation ( $\mathrm{C}, \mathrm{H}$ and $\mathrm{O}$ ), embodied in a single mixture fraction $\xi$.

One major concern in adopting the approximation in eqn (1) is the omission of any dependence on unmixedness and the high levels of scalar fluctuation, characteristic of turbulent burning; a second concern relates to the neglect of chemical reaction rates which typically vary so profoundly with temperature through Arrhenius expressions. The concentrations of reactive intermediates such as $\mathrm{CO}$ are then inaccessible.

Flamelet modelling provides a comparatively robust alternative and has also been implemented in the present simulations. Combustion is assumed to take place locally in the turbulent flame within microscopic elements which have the compositional structure of laminar flames. The latter take the form of state relationships for individual species as functions of mixture fraction 


$$
m_{\alpha}=m_{\alpha}(\xi)
$$

derived either from laminar flame measurement $[10,11]$ or computation [12]. The flowfield prediction of Favre-averaged mixture fraction mean and variance, $\widetilde{\xi}, \xi^{\prime \prime 2}$, permits the pdf $\widetilde{P}(\xi)$ to be computed in standard form - typically, a beta function. The mean composition field may then be determined by quadrature from the state relationship (3)

$$
\widetilde{m}_{\alpha}=\int_{0}^{1} \widetilde{P}(\xi) m_{\alpha}(\xi) d \xi
$$

\section{Radiation Heat Transfer}

Radiative exchange between fluid elements and the component boundaries plays an important role in the flowfield development and has been incorporated in these calculations using the discrete transfer method (DTRM). This employs a ray tracing procedure in which the radiative transfer equation is solved along paths generated by the angular discretisation of boundary cell faces. Radiative loss ( or gain ) of an individual control volume is then evaluated as a balance between emission and absorption for all rays traversing that control volume. Summation of the final intensity at the end of a line of sight in all directions yields the incident flux. For grey boundaries, the radiosity is represented by a combination of the emitted intensity and the reflected component of the incident intensity.

In recent fire source predictions in which the detailed composition and temperature structure is finely resolved, radiation properties along the non-homogeneous ray paths have been computed using the weighted sum of grey gases [13]. In the present calculations, however, in which spatial resolution is coarser and detailed experimental data for evaluation purposes is not available, a constant absorption coefficient of 0.2 , representative of combustion products at low temperature [14], has been adopted. A comprehensive review of the possible implications of implementing alternative property models is reported in [15].

Radiative exchange in the room fire simulations reported in this paper has been computed for 16 rays, fired from the solid surface of each boundary cell. This value reflects a compromise between computational economy and uniformity of coverage.

\section{Model Comparisons}

Simulations are presented which introduce systematic variation of the following model elements -

- with / without radiative exchange ( DTRM )

- heat source / eddy break-up ( EBU ) / laminar flamelet representations of the fire source

- hybrid / second-order TVD differencing schemes

- variation of mesh density - numbers of grid nodes within the compartment of 4188 , 19674 and 70432. 
Since this room configuration is symmetrical about a vertical plane bisecting the doorway and burner, only half of the room is meshed, thereby improving the flowfield resolution . Only a small number of key comparisons can be presented here but more complete information can be made available [16].

\section{COMPARISONS BETWEEN MODELS AND WITH EXPERIMENT}

A number of less detailed, but characteristic, features may be identified in the simulations. Table 1 distinguishes between predictions of the height of the neutral layer, $\mathrm{h}_{\mathrm{N}}$, ( relative to to that of the doorway, $h_{0}$ ), the respective inflow and outflow mass fluxes, and the upper layer temperature.

\section{TABLE 1}

\begin{tabular}{|l|l|l|l|l|l|c|}
\hline $\begin{array}{l}\text { Grid / } \\
\text { no. of nodes }\end{array}$ & Fire Source & DTRM & $\mathbf{h}_{\mathrm{N}} / \mathbf{h}_{0}$ & $\begin{array}{l}\text { Inflow } \\
\left(\mathrm{kg} \mathrm{s}^{-1}\right)\end{array}$ & $\begin{array}{l}\text { Outflow } \\
\left(\mathrm{kg} \mathrm{s}^{-1}\right)\end{array}$ & $\begin{array}{c}\text { Temperature } \\
\text { Upper Layer } \\
\left({ }^{0} \mathbf{C}\right)\end{array}$ \\
\hline 19674 & heat source & No & 0.557 & 0.602 & 0.603 & 145 \\
\hline 19674 & heat source & Yes & 0.514 & 0.574 & 0.575 & 122 \\
\hline 19674 & EBU & No & 0.536 & 0.627 & 0.628 & 145 \\
\hline 19674 & EBU & Yes & 0.509 & 0.578 & 0.580 & 129 \\
\hline & & & & & & \\
\hline 4188 & heat source & Yes & 0.504 & 0.592 & 0.592 & 119 \\
\hline 4188 & EBU & Yes & 0.502 & 0.594 & 0.596 & 131 \\
\hline & & & & & & \\
\hline 70432 & heat source & Yes & 0.525 & 0.623 & 0.623 & 109 \\
\hline 70432 & EBU & Yes & 0.546 & 0.521 & 0.523 & 128 \\
\hline & & & & & & \\
\hline Experiment & & & 0.561 & 0.554 & 0.571 & 129 \\
\hline
\end{tabular}

The height of the neutral layer, $h_{N}$, is defined to be the location of zero horizontal velocity within the doorway. The predicted mass fluxes, into and out of the room, are computed from the values along the mid-plane of the doorway. Their accuracy is therefore dependent upon that of the detailed velocity predictions within the doorway which is discussed later.

The table highlights a number of important issues. The neutral layer height varies most significantly with grid resolution and with the inclusion of radiative exchange, the sensitivity to fire source representation is substantially smaller. As might be expected, the introduction of radiative loss reduces both the neutral layer height and the upper layer temperature. The 
mass flux balances are generally satisfactory and again show greatest sensitivity to heat loss, although there are large differences between predicted fluxes for the two fire source descriptions with the finest grid resolution. In comparison with the experimental values, discrepancies of less than $10 \%$ are evident across a number of simulations - for the case of the finest grid, incorporating the eddy break-up combustion and discrete transfer radiation models, the discrepancies in $\mathrm{h}_{\mathrm{N}} / \mathrm{h}_{0}, \mathrm{~m}_{\text {in }}, \mathrm{m}_{\text {out }}, \mathrm{T}_{\text {upper layer }}$ are respectively $3 \%, 6 \%, 9 \%$ and $1 \%$.

In addition to chemical species prediction, which we discuss later, the introduction of a combustion model into the simulations, in preference to a simply prescribed heat source, substantially changes the structure of the fire source and plume. Energy release is then spatially distributed and coupled to the flowfield. As the computational mesh is progressively refined, this distinction becomes better defined and more influential.

Detailed comparisons with experiment are possible at two locations. Temperature measurements are reported from vertically-mounted thermocouple rakes situated in one corner, adjacent to the doorway, and within the doorway itself ( $\mathrm{cf}$. fig.1 ). At this latter position the temperature data are supplemented by velocity measurements using a bidirectional probe [1].

\section{Temperature Prediction - Corner Rake}

Figure 2 compares predictions of the vertical temperature profile in the room corner for a range of models. As indicated earlier, the introduction of radiative heat loss improves the prediction of upper layer temperature in all cases. For the intermediate grid ( 19674 internal grid nodes ), reducing the maximum temperature attained tends to deteriorate the hot layer definition as property gradients become shallower - this is particularly true in the case of the heat source representation ( $\mathrm{cf}$. fig.2a ). Distributing the heat release, through the introduction of a combustion model, appears to preserve the layered structure. Mesh refinement, in combination with a combustion model and radiative exchange, produces good agreement throughout the layer.

(a)

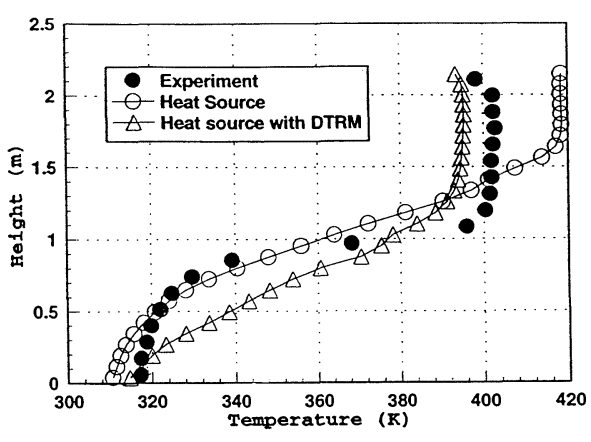

(b)

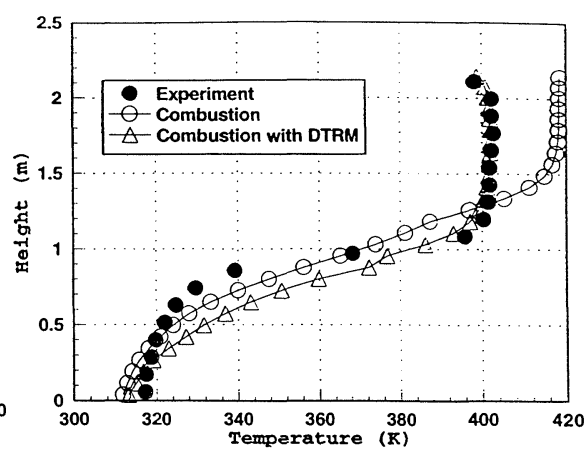

Figure 2. Vertical Temperature Profiles in the Corner of the Room : intermediate grid (a) Heat source (b) EBU Combustion Model 
Figure 3 demonstrates that the two-layer structure in the doorway is generally better defined than in the corner of the room, in both prediction and experiment. Progressive mesh refinement clearly sharpens the predicted transition between cold air inflow and dilute combustion product outflow. The DTRM simulations incorporating the EBU combustion model are again in better agreement with experiment than those involving the prescribed heat source. Replacing hybrid differencing by the TVD scheme on the intermediate grid ( 19674 nodes ) does not significantly refine the transition region. However the fine grid prediction with combustion and radiative exchange ( fig. $3 \mathrm{c}$ ) provides particularly good agreement.

(a)

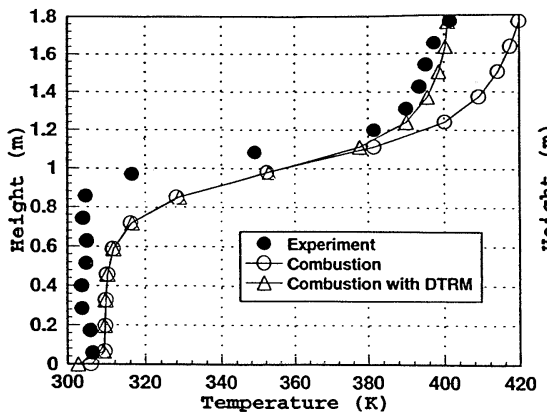

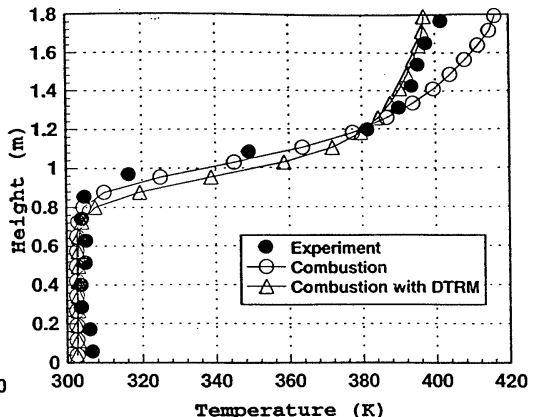

(b)

Figure 3.

Comparison of Doorway mid-plane Temperature Predictions with increasing Mesh Refinement :

(a) 4188 ; (b) $19674 ；$ (c) 70432 nodes

\section{Velocity Prediction - Doorway}

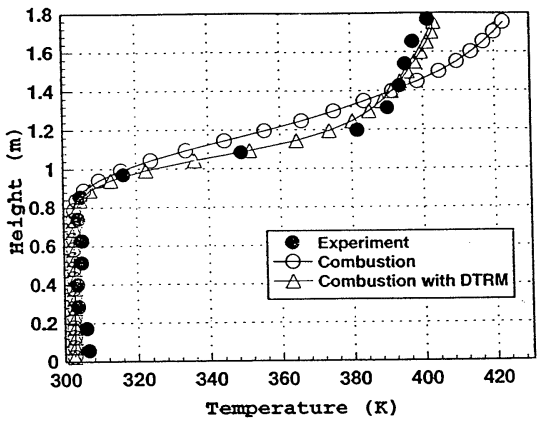

Comparisons between prediction and experiment are reported at three stations in the doorway : the horizontal velocity component in the vertical plane through the centre of the doorway and the profile across the doorway, at heights of $0.17 \mathrm{~m}$ in the cold entrant layer and at $1.65 \mathrm{~m}$ within the emergent upper layer. The predicted profiles in the vertical plane ( cf. fig. 4 ) do not appear to be strongly model sensitive. Grid resolution is revealed to be the more critical factor. The reduced neutral layer height predicted on the coarsest grid ( $\mathrm{cf}$. fig. 2a ) is accompanied by an exaggerated inflow ( fig.4a ), but the agreement is generally good.

The transverse velocity profiles have proved more controversial. In particular the measurements reveal inverted profiles in which the velocities are higher at the edge of the door jamb than in the centre. Although this might appear counter intuitive for a developed flow in a restricted passage, where wall boundary layer effects predominate, the flow in the doorway is not nearly so well-defined. Kumar et al [2], employing the JASMINE code, did not reproduce the measured trend, predicting higher velocities in the centre of the doorway, 
but for a jamb of zero depth. More recent computations [3,4] have revealed a complex set of interactions, depending upon the mesh resolution within the complete architrave.

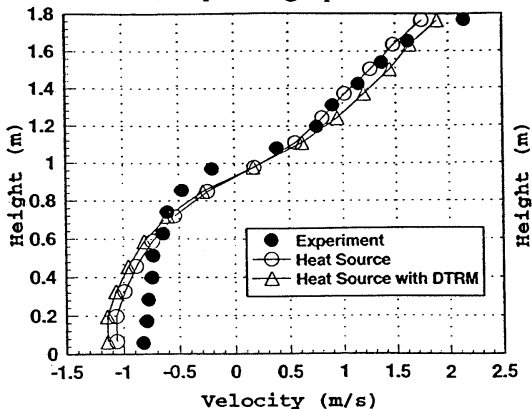

( a )

Figure 4. Comparison Between Predicted and Measured Vertical Profiles of Horizontal Velocity Component in the Doorway : (a) Heat Source with 4188 nodes ; (b) EBU Combustion Model with 19674 nodes; (c) EBU, 70432 nodes.

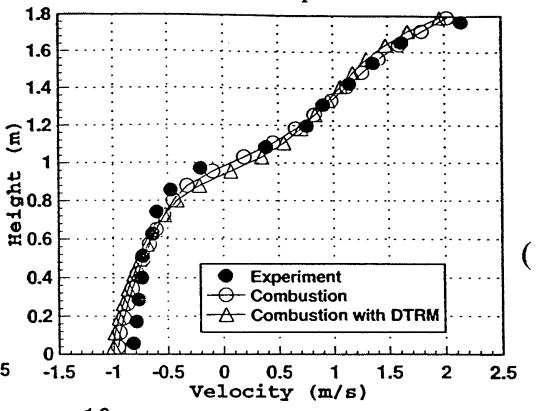

( b )

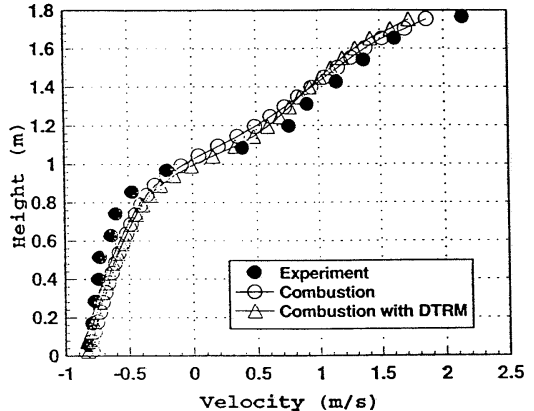

In the present calculations the depth of the door jamb is modelled by up to five cells and the evolution of the velocity profile with depth through the doorway has been computed. The velocity profiles at three planes through the doorway are illustrated in fig. 5 - moving from the room face (RP), through the jamb mid-plane (CP) to the exterior face (AP). In the upper layer ( fig.5a) the experimentally observed trend towards higher velocities closest to the door jamb is clearly reproduced in the planes closest to the room ( RP and CP ). There is however some indication of a subsequent reversal of this trend in the plane furthest from the room (AP). Such observations are broadly consistent with those reported in the earlier study [3]. The predictions in the lower layer, on the other hand, do not exhibit such behaviour. In general the computed velocities are lower adjacent to the jamb.
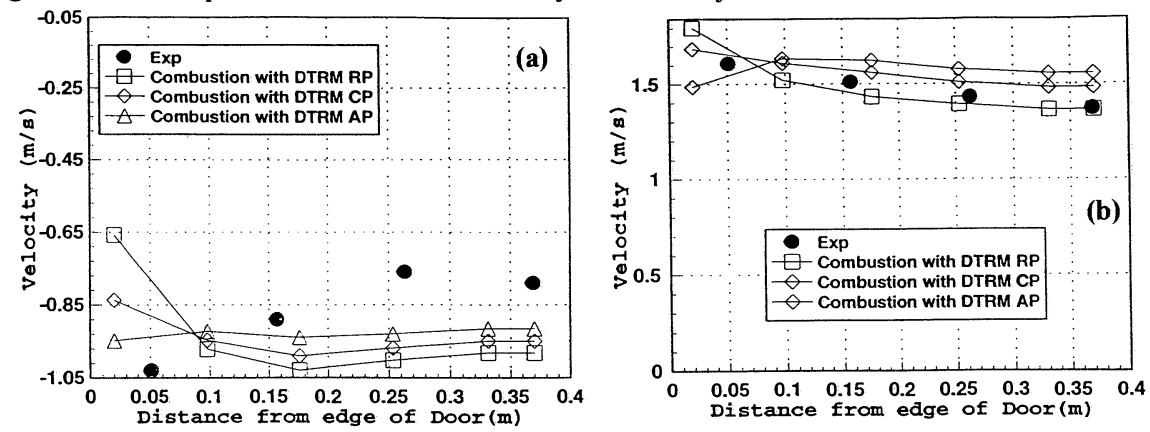

Figure 5. Predicted and Measured Profiles of Horizontal Velocity Component across the Door Jamb : (a) Lower Layer ( height $=0.17 \mathrm{~m}$ ) ; (b) Upper Layer (height $=1.65 \mathrm{~m}$ ), intermediate mesh. 
This situation is not substantially changed by further mesh refinement. Whilst the velocity levels are then brought into closer agreement with experiment, consistent with the improved mass flux predictions in Table 1 , the trend with increasing distance from the jamb remains unaffected.

\section{Chemical Species Prediction}

Whilst the comparisons reported thus far suggest that the inclusion of a combustion model offers improved predictions over those with a prescribed heat source, the absence of measured composition data in compartment fires, here and more generally, restricts the discrimination possible between alternative models. Given the ambition to better predict life threat using CFD, more detailed combustion chemistry must be incorporated however. As a first step towards broadening the predictive capabilities of codes such as SOFIE in this respect, the Steckler compartment fire has also been computed using a laminar flamelet combustion model.

The scalar state relationships employed ( cf.eqn.(3) ) are derived from small-scale laminar diffusion flame experiment [17] and, for purposes of demonstration here, these have been extended to also include the measured temperature, $T(\xi)$, and associated density, $\rho(\xi)$. In particular, by analogy with eqn.(4),

$$
\bar{T}=\bar{\rho} \int_{0}^{1} \frac{\widetilde{P}(\xi) T(\xi)}{\rho(\xi)} d \xi
$$

$$
\bar{\rho}^{-1}=\int_{0}^{1} \frac{\widetilde{P}(\xi)}{\rho(\xi)} d \xi
$$

In this case, in the absence of a computed enthalpy equation, the resulting predictions are therefore substantially adiabatic - incorporating only the small heat loss in the original laminar flame experiment. Strategies to combine detailed flamelet modelling with radiative heat loss have been developed $[13,18]$ but these are computationally more demanding and were therefore considered inappropriate to this demonstration for which comparisons with experiment are not available.
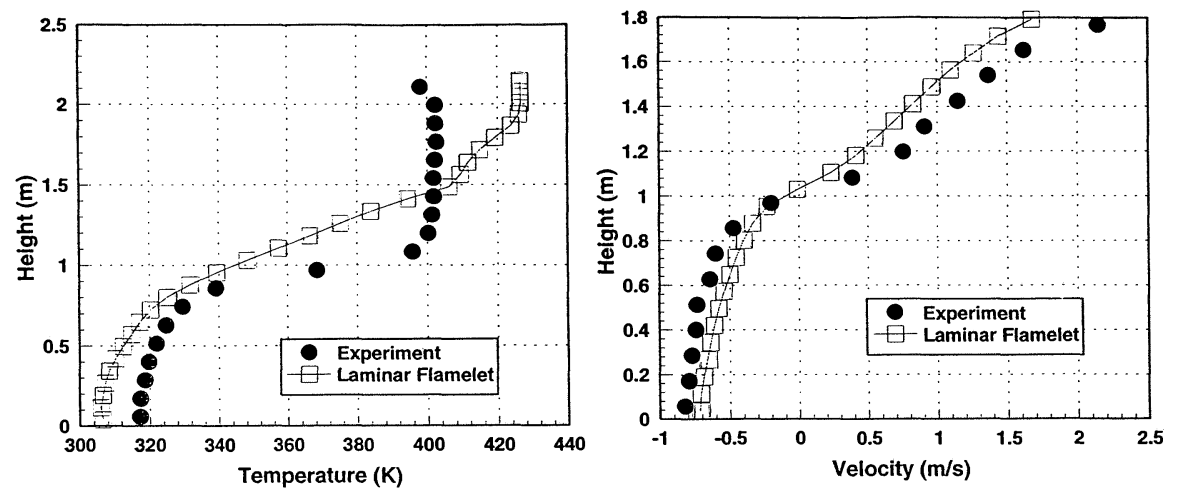

Figure 6. Comparisons of Adiabatic Flamelet Model Predictions with Experiment (a) Vertical Temperature Profile in Room Corner (b)Vertical Profile of Horizontal Velocity in Doorway. 
Illustrative profiles of the predicted room corner temperatures and mid-plane doorway velocities are shown in fig. 6 . They exhibit similar behaviour to the adiabatic predictions reported earlier in figs. $2 \mathrm{~b}$ and $4 \mathrm{~b}$. The accompanying species predictions for $\mathrm{O}_{2}, \mathrm{H}_{2} \mathrm{O}$ and $\mathrm{CO}$ are shown in fig.7. The compartment is well-ventilated and the ambient levels of $\mathrm{H}_{2} \mathrm{O}$ and $\mathrm{CO}$, remote from the fire source and near-plume regions, are very low. The predicted peak CO level ( mass fraction) in the fire is 0.026 , which compares favourably with the levels measured in other freely-burning, simulated buoyant fires of similar size [19]. For the intermediate grid employed in these predictions, however, the characteristic mesh dimension is approximately $8 \mathrm{~cm}$ and the burning envelope of the fire source is only represented by $\sim 60$ nodes. More detailed species / smoke predictions will therefore require enhanced resolution if finite rate chemistry effects are to be captured more realistically. A multi-block mesh structure, permitting local refinement of the fire source, is clearly necessary for such predictions. These computations also illustrate the requirement for more detailed composition measurements in compartment fires, embracing a wider range of ventilation conditions, in order to broaden the scope of validation studies.

(a) $\mathrm{H}_{2} \mathrm{O}$ Mass Fraction



(b) $\mathrm{O}_{2}$ Mass Fraction

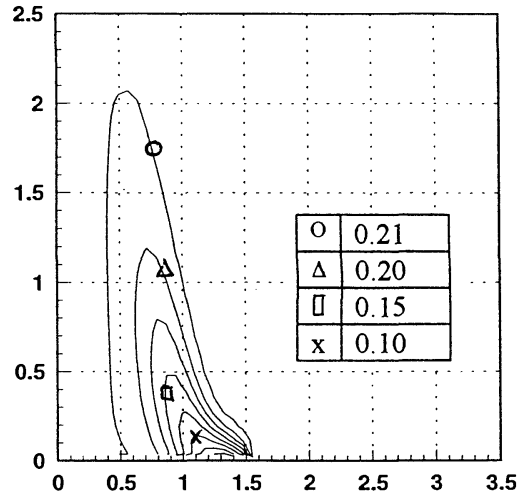

Figure 7. Flamelet Model Predictions of Species Mass Fraction in the Vertical Plane through the centre of the Fire Source and Doorway ;intermediate grid.

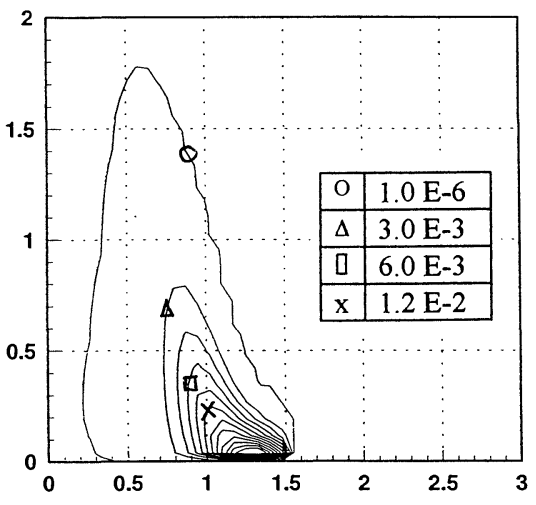

(c) CO Mass Fraction 


\section{CONCLUSIONS}

The SOFIE simulations reported demonstrate that detailed quantitative predictions of the velocity and temperature fields in compartment fires can be performed accurately, given a satisfactory combination of grid resolution and physical modelling, particularly in relation to the representations of combustion and radiative exchange.

Improved accuracy accompanies the implementation of the discrete transfer radiation model, together with eddy break-up combustion, in comparison with the adoption of a more simply prescribed heat source. In particular, the spatial distribution of heat release, accompanying the introduction of combustion models, produces significantly improved definition in the layered structure within the compartment.

The extension of combustion modelling capabilities to include laminar flamelet descriptions of turbulent burning is shown to be comparatively straightforward and preliminary predictions of reactive intermediates such as $\mathrm{CO}$ are encouraging.

\section{REFERENCES}

[1] Steckler,K.D.,Quintiere,J.G. and Rinkinen,W.J. (1984) Flow Induced by Fire in a Compartment, NBSIR 82-2520, Nat.Bureau of Standards, Washington DC.

[2] Kumar,S., Gupta,A.K. and Cox,G. (1991) Effects of Thermal Radiation on the Fluid Dynamics of Compartment Fires, Proc. Third Int.Symp. on Fire Safety Science,Elsevier,p.345.

[3] Kerrison,L., Galea,E.R., Hoffman,N. and Patel,M.K. (1994) A Comparison of a FLOW3D Based Fire Field Model with Experimental Room Fire Data, Fire Safety Journal $\underline{23}, 387$.

[4] Mawhinney,R.N., Galea,E.R., Hoffman,N. and Patel,M.K. (1994) A Critical Comparison of a PHOENICS Based Fire Field Model with Experimental Compartment Fire Data, J.Fire Prot.Engr. $\underline{6}(4), 137$.

[5] Kerrison,L.,Mawhinney,N., Galea,E.R., Hoffman,N. and Patel,M.K.(1994) A Comparison of Two Fire Field Models with Experiemntal Room Fire Data, Proc. Fourth Int.Symp. on Fire Safety Science, IAFSS, p.13.

[6] SOFIE Users Manual, ed. Rubini,P.A. (1996) School of Mechanical Engineering, Cranfield University,UK.

[7] Magnussen,B.F. and Hjertager,B.H. (1976) On Mathematical Modelling of Turbulent Combustion with Special Emphasis on Soot Formation and Combustion, Sixteenth Symp. (Int.) on Combustion, Combustion Institute, p.719.

[8] Liew,S.K., Bray,K.N.C. and Moss, J.B. (1984) A Stretched Laminar Flamelet Model of Turbulent Nonpremixed Combustion $\underline{56}, 199$. 
[9] Lockwood,F.C. and Shah,N.G. (1981) A New Radiation Solution Method for Incorporation in General Combustion Prediction Procedures, Eighteenth Symp.(Int.) on Combustion, Combustion Institute, p.1405.

[10] Crauford,N.L., Liew,S.K. and Moss, J.B. (1985) Experimental and Numerical Simulation of a Buoyant Fire, Combust.Flame 61, 63.

[11] Sivathanu, Y.R. and Faeth,G.M. (1990) Generalised State Relationships for Scalar Properties in Nonpremixed Hydrocarbon-Air Flames, Combust.Flame 82, 211.

[12] Warnatz,J., Maas,U. and Dibble,R.W. (1995) Combustion, Springer.

[13] Bressloff,N.W., Moss,J.B. and Rubini,P.A. (1996) CFD Prediction of Coupled Radiation Heat Transfer and Soot Production in Turbulent Flames, Twenty-Sixth Symp.(Int.) on Combustion, ( to be presented ).

[14] Modest, M.F. ( 1993 ) Radiative Heat Transfer, McGraw-Hill.

[15] Bressloff,N.W., Moss, J.B. and Rubini,P.A. (1996) Assessment of a Differential Total Absorptivity Solution to the Radiative Transfer Equation as applied in the Discrete Transfer Radiation Model , Numerical Heat Transfer : Part B Fundamentals, 29(3), 381.

[15] Lewis,M.J. (1996) SOFIE Computations of the Steckler Compartment Fire, SOFIE Report 01/96, Cranfield University,UK.

[16] Mitchell,R.E., Sarofim,A.F. and Clomburg,L.A.(1980) Experimental and Numerical Investigation of Confined Laminar Diffusion Flames, Combust.Flame 37, 227.

[17] Young,K.J. and Moss,J.B. (1995) Modelling Sooting Turbulent Jet Flames using an Extended Flamelet Technique, Combust. Sci. Tech. 105, 33.

[18] Smith,D.A. and Cox,G. (1992) Major Chemical Species in Buoyant Turbulent Diffusion Flames, Combust.Flame 91, 226.

\section{Acknowledgement}

The authors are pleased to acknowledge the financial support of EPSRC through research grant GR/J71540 and of the international Consortium managing the development of the SOFIE code at Cranfield. 